\title{
La formación para la investigación de los profesionales de la información en la universidad española
}

\author{
Vivina Asensi-Artiga \\ Departamento de Información y Documentación \\ Universidad de Murcia
}

\subsection{Resumen}

A partir de la experiencia de la Universidad de Murcia, se analiza el contexto, los objetivos y los condicionantes de la formación para la investigación en cada uno de los ciclos de las enseñanzas universitarias - diplomatura, licenciatura y doctorado- y con especial atención a las Ciencias de la Documentación. Se concluye que los conocimientos en materia de investigación científica son necesarios para todos los profesionales de la información, que el método científico es válido como pauta a seguir en todas las tipologías de trabajo intelectual, y que la formación de investigadores exige un proceso de reflexión en cuanto a la naturaleza, objetivos y elementos de la ciencia.

Palabras clave: Universidad. España. Formación en la investigación. Diplomaturas. Licenciaturas. Doctorados. Ciencias de la Documentación. Técnicas del trabajo científico.

\subsection{Abstract}

The context, aims and prerequisites of the education for research - with an emphsis in Information and Documentation - are analysed, with a focus in the contribution done by —and to be done in - each of the university cycles — graduate, postgraduate and doctoral-, from the perspective and experience of the University of Murcia. It is concluded that knowledge of scientific research methodology is necessary for all the information professionals, that the scientific method is valid for any kind of intellectual work, and that researchers' instruction requires a process of reflection on the nature, purpose and elements of science.

Keywords: University. Spain. Education for research. Graduate studies. Postgraduate studies. Doctoral studies. Library and Information Science. Scientific research techniques. 


\section{Introducción}

En España, la formación académica de investigadores y de otros profesionales de la información que desarrollan su trabajo al servicio de los investigadores, corresponde a la Universidad. A pesar de que otras instituciones desarrollan tareas de formación para la investigación, es competencia de la Universidad la planificación de las enseñanzas y la expedición de las titulaciones correspondientes en los tres ciclos previstos.

En la actualidad, el diseño curricular que ofrece la Universidad, permite la formación para la investigación a diferentes niveles, de acuerdo con los objetivos de los planes de estudio correspondientes, enmarcados en cada uno de los ciclos de la enseñanza universitaria.

Concretamente, las titulaciones relacionadas con la Documentación, Diplomatura en Biblioteconomía y Documentación y Licenciatura en Documentación, constituyen un claro exponente de la oportunidad de la formación en esta materia de los futuros profesionales, que realizarán su trabajo al servicio de la documentación. Las diferentes materias que componen cada uno de los ciclos de la enseñanza universitaria, aportan contenidos que se pueden interrelacionar y orientar al servicio de la investigación, aunque no es su objetivo exclusivo.

Lógicamente, los objetivos de los planes de estudio de los dos ciclos de la enseñanza superior indican ya el nivel y la formación de los profesionales que culminan esta formación. Si tenemos en cuenta las directrices generales comunes de los planes de estudio de carácter oficial, con validez en todo el territorio nacional (1), y considerando las tareas propias de estos titulados en el campo laboral, no es difícil programar la formación sobre y para la investigación científica. Bibliotecarios y documentalistas poseen la formación generalista adecuada para servir a todo tipo de usuarios; sin embargo, la diferente cualificación de diplomados y licenciados implica el desarrollo de su actividad profesional desde diferentes grados de especialización.

Respecto a los estudios de tercer ciclo, la formación en las técnicas de investigación científica es materia obligada durante el periodo de docencia de los programas de doctorado (2). Incluso los licenciados en Documentación, que ya han estudiado con detenimiento las técnicas documentales aplicadas a la investigación, deben profundizar en el estudio del método científico y de todos los elementos que conforman la naturaleza de la Ciencia; es decir, en todas las etapas del proceso de investigación científica, en la correcta aplicación del método general de la ciencia, y en el conocimiento de las técnicas, generales o específicas, necesarias para la consecución de los objetivos del tercer ciclo: la exposición y defensa de la Tesis Doctoral, que permite obtener la máxima titulación académica: el grado de Doctor. 
Es nuestro objetivo el estudio del entorno relacionado con la formación en investigación científica, basándonos como punto de partida en la experiencia de la Universidad de Murcia, partiendo de las posibilidades ofertadas por el Departamento de Información y Documentación y, desde su creación, por la Facultad de Ciencias de la Documentación.

\section{Material y método}

Para llevar a cabo nuestros objetivos, hemos consultado los planes de estudio de las titulaciones ofertadas en nuestra área y hemos sometido a examen los resultados obtenidos a través de nuestra experiencia en la formación de los alumnos de primero y segundo ciclo de nuestra universidad, así como de los alumnos de tercer ciclo de la Universidad de Murcia y de la Universidad Politécnica de Valencia.

La experiencia con alumnos, a través de varias promociones, constituye un material de valor incalculable para examinar con espíritu crítico y reflexivo sus necesidades prioritarias así como las deficiencias observadas y que, sin duda alguna, obedecen a diversos factores dependientes de los diferentes elementos implicados en el proceso enseñanza-aprendizaje. Por otra parte, la legislación vigente nos permite recapacitar y no perder de vista los objetivos para los que se diseñaron los tres ciclos de la enseñanza universitaria.

\section{Estudios de primer ciclo: Diplomatura en Biblioteconomía y Documentación}

La sociedad en su estructura de puestos de trabajo contempla y exige la presencia de profesionales de la información capacitados para atender a diferentes tipos de usuarios que demandan información general sobre temáticas diversas, tanto en los servicios públicos como privados. La formación de los diplomados en Biblioteconomía y Documentación responde a estas necesidades y, mediante los contenidos de las materias optativas y de las asignaturas de libre configuración, permiten profundizar en mayor medida en determinados contenidos para completar la formación.

Iniciarse en el conocimiento del método científico no es tarea inútil ni excesiva para un primer ciclo, siempre que se tenga como punto de referencia los objetivos de los planes de estudio y el nivel de conocimientos que ha de superar el alumno.

Ciertamente, durante la diplomatura el alumno adquiere amplia formación en cuanto al análisis documental, a las fuentes de información, y se inicia en el conocimiento de las Tecnologías de la Información, alcanzando un nivel suficiente para proseguir su formación en este campo y beneficiarse de las innovaciones que continuamente nos ofrecen.

Scire. $7: 2$ (jul.-dic. 2001) 39-52. 
Por otra parte, antes de finalizar sus estudios tiene que superar el practicum. La evaluación de esta asignatura exige la presentación de una memoria sobre las actividades realizadas, para lo cual tendrá que utilizar una metodología rigurosa, acorde con la formación académica recibida.

En este contexto curricular, algunas universidades ofrecen asignaturas optativas que se plantean como objetivo la formación de los alumnos en cuanto a la adopción del método científico como método habitual de trabajo (Eco, 1993), el conocimiento de las necesidades del investigador, la diferenciación de los diversos tipos de trabajo (investigación, divulgación, etc.), así como los tipos de documentos que se generan tras el proceso de comunicación de los resultados obtenidos o a consecuencia de la petición para la subvención de un proyecto determinado.

Por tanto, parece adecuado que la programación de esta materia, o de esta asignatura, considere incluir entre sus contenidos unos conocimientos generales en relación con el conocimiento científico y las actividades que deberá desempeñar como elemento del proceso de investigación, una formación específica que le permita aplicar los conocimientos adquiridos en la diplomatura al servicio de los investigadores, y, por último, unos conocimientos metodológicos acerca de la comunicación científica.

En la primera parte del programa se abordan los conocimientos generales:

- Introducción al concepto de Ciencia, como marco del proceso de investigación científica.

- Características del método científico, con el objetivo de estudiar con mayor detenimiento las etapas del proceso de investigación científica y diferenciar los tipos de técnicas adecuadas para cada una de ellas.

- Estudio del protagonismo del documentalista en el proceso de investigación científica. Al mismo tiempo, el alumno debe tener conocimientos de ética relacionados con su actividad profesional, al servicio del investigador.

Respecto a la formación específica, que le permitirá aplicar sus conocimientos al servicio de la investigación, no resulta difícil diseñar las lecciones de manera que el alumno consiga relacionar dichos conocimientos con su utilidad como técnicas de investigación.

En consecuencia, la segunda parte del programa,corresponderá a:

- Breve resumen de los principales campos de investigación en el Área de Biblioteconomía y la Documentación y características específicas del proceso de investigación científica en este campo.

- Acceso a la información, en la fase de documentación del proceso de investigación científica. El alumno debe conocer las necesidades del investigador, tanto para recopilar información sobre el conocimiento precedente 
relacionado con el problema que pretende resolver (3), como sobre las convocatorias para la concesión de becas o proyectos, así como para recuperar toda la información necesaria para su participación en dichas convocatorias. La aplicación de las Tecnologías de la Información es imprescindible para la consecución de estos objetivos.

- Importancia del Análisis Documental para la recuperación de la información, haciendo especial hincapié en la normalización de los puntos de acceso a los registros. En este apartado, se insiste en la importancia del documentalista como intermediario entre el usuario - que se expresa en el lenguaje propio de su trabajo - y el lenguaje utilizado por el sistema de recuperación que se va a consultar, ya que es necesario traducir el lenguaje natural del investigador (aunque sea un lenguaje especializado) al lenguaje documental.

Por último, la tercera parte del programa, está destinada a establecer una metodología de trabajo en cuanto al tratamiento de la documentación obtenida en la primera etapa del proceso, la estructura de los trabajos de investigación y a su presentación, mediante la comunicación oral o escrita. Consta en concreto de las siguientes unidades:

- Técnicas Documentales, considerando las técnicas de lectura, la elaboración de documentos de trabajo (mediante aplicaciones informáticas).

- Elementos comunes de la Comunicación Científica, considerando al mismo tiempo las diferentes formas de comunicación. Es fundamental que los alumnos conozcan la estructura de un trabajo científico o técnico, tanto para trabajar correctamente desde sus inicios en este campo, como para su tratamiento documental. El aprendizaje de la confección y posterior comunicación de un trabajo científico o técnico, aplicando el método científico, es necesaria para conseguir estructurar la mente de manera que la aplicación del método se convierta en una función habitual siempre que realicemos estas actividades.

- El lenguaje administrativo, como medio imprescindible para la concurrencia a programas de financiación de proyectos e incluso de actividades profesionales. De este modo, se explica la redacción de una instancia, los contenidos de un curriculum vitae, o la confección de informes.

Los alumnos que han optado por esta materia, deben tener claros los sigueintes objetivos:

- Conocer el entorno del investigador y la ética del documentalista en relación con la investigación.

- Concienciarse del protagonismo que el profesional de la información alcanza en el entorno de la Ciencia. 
- Iniciarse en el conocimiento del lenguaje relacionado con la documentación científica y técnica.

- Adquirir una metodología de trabajo en el campo de la Ciencia y de la Tecnología.

Por otra parte, es conveniente que los alumnos comprendan que los conocimientos asimilados en otras materias, o en otras asignaturas, son importantes y tienen su razón de ser en cuanto que están al servicio al usuario, y desde qué punto de vista son útiles para proporcionar información indispensable para los investigadores.

\section{Estudios de Segundo Ciclo: Licenciatura en Documentación}

Las directrices de la Licenciatura en Documentación (4), establecen que dichas enseñanzas proporcionarán una formación adecuada en los aspectos básicos y aplicados de la información y la documentación científica y especializada, del tratamiento y recuperación de la información y en los aspectos de la planificación, organización y gestión de los correspondientes sistemas, redes y centros.

La Licenciatura en Documentación no son estudios de segundo ciclo, prácticamente obligatorios para los Diplomados en Biblioteconomía y Documentación; en efecto, no constituye una continuación de la Diplomatura en Biblioteconomía y Documentación. De hecho cada vez más los alumnos de la Licenciatura proceden de otras titulaciones y obtienen excelentes resultados académicos. Además, entre las universidades españolas, en unas está implantada sólo la Diplomatura, en otras sólo la Licenciatura, y en otras, ambas titulaciones.

Ambos estudios son muy recientes, sin embargo, la licenciatura carece de la tradición de la diplomatura. Ciertamente, el diseño curricular de la Diplomatura - cuyos planes de estudio fueron aprobados por el Ministerio de Educación y Ciencia en 1981(5) — tiene su precedente en el de la Escuela de Biblioteconomía adscrita a la Diputación Provincial de Barcelona (6). Sin embargo, la Licenciatura no tiene raíces semejantes. Son estudios sin tradición universitaria, diseñados para satisfacer la necesidad social actual de profesionales de la información especialistas. En consonancia, las materias que componen el curriculum según las directrices oficiales de sus planes de estudio están adscritas a las diversas áreas del conocimiento.

Estas características han propiciado en cada Universidad un enfoque diferente en cuanto a contenidos y diseño curricular, de las asignaturas que conforman las materias establecidas. No obstante, de una manera general podemos aportar en este trabajo la formación en investigación propia de estos estudios.

Los futuros licenciados en Documentación, por su formación, ocuparán puestos de responsabilidad en servicios de información y documentación; por 
tanto, las materias troncales están diseñadas con estos objetivos. A partir de ahí, los alumnos pueden poner al servicio de la investigación los conocimientos adquiridos las asignaturas dedicadas a la "Estadística", "Técnicas de Indización y Resumen en Documentación Científica", "Técnicas Documentales aplicadas a la investigación", y "Planificación y Evaluación de Sistemas de Información y Documentación".

En cuanto a la materia troncal "Técnicas documentales aplicadas a la investigación", de acuerdo con las directrices establecidas, sus contenidos corresponden al estudio de técnicas estadísticas, analíticas y descriptivas de la investigación, así como al conocimiento de la investigación en documentación.

Además, los alumnos pueden aprovechar para completar su formación para la investigación asignaturas optativas, como "Investigación Operativa", "Documentación Técnica y Literatura Gris", "Legislación en Información y Documentación".

De este modo, los contenidos de estas materias y de las asignaturas optativas mencionadas incluyen:

- Técnicas estadísticas aplicadas a la documentación científica y a la investigación científica en general.

- Diseño y gestión de sistemas de indización y de resumen, aplicados a los documentos científicos.

- Evaluación de los sistemas de información, teniendo en cuenta las necesidades de los investigadores.

- Aplicación y análisis de las técnicas adecuadas para la investigación documental, considerando las últimas tendencias en cuanto a las posibilidades que ofrecen las Tecnologías de la Información.

- El conocimiento de las principales tendencias de investigación en el campo de la Documentación es necesario para que los alumnos conozcan y comprendan las principales cuestiones planteadas en él, valoren su importancia, crezca su interés por ellas, $\mathrm{y}$, al menos, se conciencien de que tienen que permanecer alerta para conocer cuantas innovaciones se produzcan.

- Conocimientos para la planificación, programación y control de proyectos, puesto que el alumno debe superar la evaluación de un proyecto, antes de finalizar sus estudios de Licenciatura.

- Además de profundizar en el estudio de la literatura gris y de su problemática actual, es necesario que el profesional conozca en profundidad las características de la Documentación Técnica. La metodología para la realización de informes científicos y técnicos, tanto respecto a su estructura como a su contenido, le será de utilidad para confeccionar su proyecto y para la recupe-

Scire. $7: 2$ (jul.-dic. 2001) 39-52. 
ración de esta tipología documental. En realidad, el estudio de la problemática que encierra este tipo de documentos, sobre todo en cuanto a la aplicación del proceso documental normalizado, constituye una novedad en el campo del análisis formal, puesto que aún no se ha consolidado suficientemente.

En este segundo ciclo, por tanto, el profesional de la información recibe la formación suficiente para la aplicación de las técnicas de documentación a la investigación científica, y alcanza los conocimientos necesarios para su actividad profesional al servicio de la investigación.

La universidad dedica a la formación de investigadores un nuevo nivel educativo, las enseñanzas de tercer ciclo, que conducen a la obtención del título de doctor, máxima titulación académica, que confiere la plena capacidad investigadora.

\section{Los estudios de tercer ciclo: Los programas de doctorado}

A pesar de su juventud — dos o tres años de implantación dependiendo de las universidades - la reforma de los estudios de doctorado, al permitir el desarrollo de programas de doctorado interdepartamentales e incluso interuniversitarios, está consolidando la participación de los profesores del área de Biblioteconomía y Documentación en programas pertenecientes a otras áreas del conocimiento. La demanda de esta colaboración se produce en aquellos campos relacionados con la investigación que han aplicado el método científico con mayor rigor a sus investigaciones y que, en consecuencia, fueron pioneros en valorar la importancia de la documentación y de todo lo relacionado con la recuperación de la información.

Por otra parte, el crecimiento en cuanto a la oferta de titulaciones (licenciaturas) así como la conversión en titulaciones universitarias de otros estudios dependientes de otros organismos — como por ejemplo los conservatorios, institutos de educación física - ha impulsado el nacimiento de nuevos programas de doctorado adaptados a sus necesidades o a la admisión de estos titulados en los programas ya existentes. Así, en la Universidad de Murcia, se cuenta con programas de doctorado en los que se indica como titulo de entrada: Licenciado en Educación Física; en la Universidad Politécnica de Valencia, se admite en el curso de doctorado de Técnicas y Métodos Actuales en Información y Documentación a los titulados del Conservatorio Superior de Música, así como a los Licenciados en Bellas Artes, puesto que el Área de Biblioteconomía y Documentación, administrativamente pertenece al Departamento de Comunicación Audiovisual, Documentación e Historia del Arte .

Esta reforma, al limitar el número de créditos que pueden ofertar los diferentes programas, así como el número de cursos por departamentos, ha propiciado la optimización de recursos y un nuevo planteamiento en el diseño de los programas. 
Nuestra experiencia en cuanto a las enseñanzas de tercer ciclo (Anexo I) se inicia en el bienio 1992-94, cuando por primera vez participamos en la docencia de los programas de doctorado, concretamente en el programa Problemas y métodos actuales de la Lingüística Española, del Departamento de Lengua Española y Lingüística General. En este programa participamos hasta el bienio 1994-1996.

Durante el bienio 1994-1996 el Departamento de Información y Documentación de la Universidad de Murcia ofertó su propio Programa de Doctorado, dentro del Área de Humanidades, aunque el título final de Doctor que obtenían los alumnos era el de Doctor en Letras (Filología Hispánica), porque no se había implantado la Licenciatura en Documentación y la Comisión de Doctorado de la Universidad estimó que éste era el título más próximo de los disponibles de acuerdo con la Nomenclatura Internacional de la UNESCO.

En la actualidad, participamos en programas de doctorado pertenecientes a las Áreas de Ciencias de la Salud, Humanidades y ciencias Sociales. Es evidente que el punto de partida para formación en cuanto a investigación científica se refiere es distinto según la titulación de los alumnos. De todas manera, no olvidemos que la formación de investigadores corresponde al tercer ciclo. Por tanto, se les ha de proporcionar unos conocimientos profundos de todos los elementos que conforman el proceso de investigación, así como de su razón de ser y de sus objetivos. Los alumnos, incluso los procedentes de la licenciatura en Documentación, poseen conocimientos parciales, pero nuestra experiencia nos permite afirmar que necesitan profundizar en el estudio de la metodología de la investigación científica.

A continuación se enumeran las características que presentan los alumnos procedentes de las diferentes titulaciones:

- Los alumnos de Documentación, poseen conocimiento de las técnicas documentales, así como de las principales líneas de investigación en el campo de la documentación.

- Los alumnos procedentes de Medicina poseen conocimientos de documentación. Sus planes de estudio incluyen materias relacionadas con la Historia de la Medicina y la Documentación Médica. Téngase en cuenta que, aparte de ello, existe bibliografía en la actualidad tanto relacionada con la metodología científica (que incluso es utilizada para otras disciplinas), como sobre el uso de Internet. Además existen cursos puntuales sobre el uso de Medline, etc., que imparten en sus lugares de trabajo (hospitales, etc.)

- Los alumnos procedentes de Química y Biología poseen conocimientos relacionados con algunas bases de datos concretas que están utilizando en su actividad investigadora. Algunos de ellos son becarios de investigación y se están iniciando en la recuperación de información. 
- Los alumnos procedentes de Humanidades no tienen formación en metodología científica ni en las técnicas de investigación.

- Los alumnos procedentes de Plástica, Música y Educación Física poseen aptitudes y gran interés por la recuperación de la información. En cuanto al método científico, son muy receptivos a formarse en su conocimiento para su aplicación práctica.

Por tanto, es conveniente que todo programa relacionado con el método científico, incluya unos contenidos generales respecto a la Ciencia y los elementos que conforman su naturaleza. Esto no es incompatible, antes al contrario, con que el estudio de las técnicas específicas sea el adecuado para cada área del conocimiento. Así, los alumnos reciben enseñanzas acerca de los sistemas de información que les permitirán aplicar estas técnicas documentales a su investigación específica, capacitándoles para aprovechar y beneficiarse de las prestaciones que puedan ofrecerles los avances tecnológicos.

Partiendo de estas consideraciones, en la programación de esta materia se deberían incluir los siguientes contenidos:

- Concepto y objetivos de la investigación científica, reflexionando sobre la formación del investigador y las características y objetivos de los estudios de tercer ciclo. Al mismo tiempo, conviene ofrecer una visión de conjunto sobre la estructura de la Universidad, para darle a conocer el marco en que se desarrolla la investigación.

- Estudio teórico-práctico del método general de la Ciencia y de todo el proceso de investigación científica, recurriendo a la bibliografía especializada. El alumno deberá conocer las Técnicas generales del trabajo de investigación, haciendo en este aspecto especial hincapié, porque es a las que menos importancia les concede, cuando son fundamentales para llevar a cabo una investigación rigurosa. Tanto la formulación de la hipótesis como su verificación, son facetas que el alumno debe tener en cuenta, de manera que el pensamiento reflexivo presida toda su actividad intelectual.

- Técnicas de trabajo intelectual, que son fundamentales para la creación y comunicación del conocimiento científico. Por tanto, las operaciones propias de la inteligencia, para formular argumentos y proposiciones, y para dar a conocer el resultado del problema planteado (el resultado de la investigación) deben ser asimiladas con sencillez, pero con claridad.

- Planteamiento del problema de investigación y organización del trabajo. Se tendrá en cuenta tanto la fase exploratoria y las consideraciones a tener en cuenta para la elección del tema, siguiendo unas pautas previamente establecidas. Por último, dentro de este apartado general se estudiará la problemática que supone la delimitación del campo de actuación. 
- La etapa de documentación, el acceso a las fuentes y las técnicas documentales. Son contenidos en los que es necesario prestar especial atención a los alumnos dependiendo de la licenciatura de la cual proceden. Sin embargo, se hace necesario insistir, en general, en la importancia de esta etapa y en la aplicación de las técnicas precisas para obtener los máximos beneficios de los servicios que están a su alcance.

- Elaboración y presentación de los trabajos de investigación, y las características propias de la Tesis Doctoral. Debe respetarse, dentro de la metodología y las normas establecidas, las idiosincrasia propia de las diferentes áreas del conocimiento. Es fundamental que los futuros investigadores, se inicien en su actividad científica con la humildad propia del científico que nunca cree estar en posesión de la verdad exclusiva, sin que por ello renuncie a los resultados de sus investigaciones. Del mismo modo es necesario respetar la tradición establecida en cuanto a formas de presentación y comunicación del trabajo realizado.

- Fuentes de información sobre las principales convocatorias de financiación de proyectos de investigación y directrices comunitarias.

No obstante, debemos insistir en que el alumno debe aprender a deducir, a partir de los fundamentos teóricos de la Ciencia y todo lo relacionado con la investigación científica, tal como viene reflejado en la bibliografía, la aplicación práctica y actual de dichos contenidos.

Las bases teóricas son válidas para sentar las bases fundamentales, pero hay que saber llevar el trabajo a la práctica, respetando el método científico. El objetivo en cuanto a la formación de los alumnos de doctorado es que consigan llevar a cabo la aplicación práctica del método científico a su trabajo de investigación.

Cuando un alumno pregunta dudas sobre las cuestiones teóricas, siempre espera una respuesta con aplicación práctica para su trabajo: Hemos de tener en cuenta que normalmente nuestros alumnos no se dedican a la filosofía de la Ciencia.

\section{Conclusiones}

- Los conocimientos en materia de investigación científica son necesarios para todos los profesionales de la Información.

- El método Científico es válido como pauta a seguir en todas las tipologías de trabajo intelectual.

- La formación de investigadores exige un proceso de reflexión, en cuanto a la naturaleza, objetivos y elementos que conforman la naturaleza de la Ciencia. 


\section{Notas}

(1) Real Decreto 1497/1987, de 27 de noviembre, (B.O.E. 14 de diciembre de 1987) por el que se establecen las directrices generales comunes de los planes de estudio de los títulos de carácter oficial y validez en todo el territorio nacional. El Artículo $3^{\circ}$ indica la duración y ordenación cíclica de las enseñanzas: 1. Las enseñanzas universitarias conducentes a la obtención de los títulos oficiales a que se refiere el artículo primero se estructurarán en ciclos de acuerdo con lo dispuesto en la Ley de Reforma Universitaria (R. 1983, 1856 y Ap. 1975-85, 13793) y en este Real Decreto. 2. El primer ciclo de las enseñanzas universitarias comprenderá enseñanzas básicas y de formación general, así como en su caso, enseñanzas orientadas a la preparación para el ejercicio de actividades profesionales. El segundo ciclo estará dedicado a la profundización y especialización en las correspondientes enseñanzas, así como a la preparación para el ejercicio de actividades profesionales.

(2) De acuerdo con la legislación vigente, Real Decreto 778/1998, de 30 de Abril, (BOE 1 de Mayo de 1998): "Los estudios de tercer ciclo conducentes a la obtención del título de Doctor [...] tendrán como finalidad la especialización del estudiante en un campo científico, técnico o artístico determinado, así como su formación en las técnicas de investigación [...] en orden a la presentación y lectura de la correspondiente tesis doctoral".

(3) El proceso de investigación se inicia cuando surge un problema del que no se sabe la solución.

(4) Real Decreto 912/1992, de 17 de julio de 1992 (B.O.E. 27 de agosto de 1992).

(5) Orden de 24 de Febrero de 1981 (B.O.E. 9 de Enero de 1979).

(6) En 1915 se creó la Escuela Superior de Bibliotecarias, que a partir de 1974 y hasta 1982 fue la Escuela de Bibliología. En 1982 se convirtió en E.U. de Biblioteconomía y Documentación, adscrita a la Diputación Provincial de Barcelona, hasta 1997.

\section{Referencias}

Carreras Panchon, A. (Coor.) (1994). Guía práctica para la elaboración de un trabajo científico. Salamanca : Universidad, 1994.

Costa Carballo, C. M. da (1996). Introducción a la información y documentación médica. Barcelona : Masson, 1996.

Desantes Guanter, J. M ${ }^{\text {a }}$; López Yepes, J. (1996). Teoría y Técnica de la Investigación Científica. Madrid : Síntesis, 1996.

Eco, U. (1997). Cómo se hace una Tesis. Barcelona : Gedisa, 1997.

Lakatos, E. Ma ; Marconi, M. de Andrade (1992). Metodologia do trabalho científico: procedimentos básicos, pesquisa bibliográfica, projeto e relatorio, publicaçoes e trabalhos científicos. $4^{\mathrm{a}}$ ed. São Paulo: Atlas, 1992.

López Yepes, J. (1995). La aventura de la investigación científica. Madrid : Síntesis, 1995. Pádua, E. M. Marchesini de (1997). Metodologia da pesquisa. $2^{a}$ ed. Campinas. S.P.: Papirus, 1997 
Rudio, F. V. (1986). Introdução ao projeto de pesquisa científica. 24 a ed. Petrópolis: Vozes, 1986

Sierra Bravo, R. (1996). Tesis Doctorales y trabajos de Investigación Científica. $4^{\mathrm{a}}$ ed. Madrid, Paraninfo, 1996

Tapia, L. E. Rodríguez (1999). Elaboração de projectos de investigação científica: guia para pesquisadores em formaçao inicial e avançada. São Paulo: Cid Editora, 1999

Thiollent, M. (1998). Metodologia da pesquisa-açao. $8^{\text {a }}$ ed. São Paulo: Cortez, 1998.

\section{Anexos}

\section{Anexo I. Programas Doctorado al amparo del Real Decreto 185/1985}

\begin{tabular}{|c|c|c|c|c|}
\hline Bienio & Departamento & Programa & $\begin{array}{l}\text { Procedencia } \\
\text { Alumnos }\end{array}$ & $\begin{array}{l}\text { Título finales } \\
\text { del programa }\end{array}$ \\
\hline $1992 / 94$ & $\begin{array}{l}\text { Lengua Española y } \\
\text { Lingüística General }\end{array}$ & \multirow{3}{*}{$\begin{array}{l}\text { Problemas y métodos } \\
\text { actuales de la } \\
\text { Lingüística Española }\end{array}$} & \multirow{3}{*}{$\begin{array}{l}\text { Licenciados en } \\
\text { Filosofía y Letras } \\
\text { y/o Letras }\end{array}$} & \multirow{5}{*}{$\begin{array}{l}\text { Doctor en } \\
\text { Filosofía y } \\
\text { Letras (Filología } \\
\text { Hispánica) }\end{array}$} \\
\hline $1993 / 95$ & $\begin{array}{l}\text { Lengua Española y } \\
\text { Lingüística General }\end{array}$ & & & \\
\hline $1994 / 96$ & $\begin{array}{l}\text { Lengua Española y } \\
\text { Lingüística General. }\end{array}$ & & & \\
\hline & $\begin{array}{l}\text { Información y } \\
\text { Documentación }\end{array}$ & \multirow{2}{*}{$\begin{array}{l}\text { Técnicas y Métodos } \\
\text { actuales en } \\
\text { Información y } \\
\text { Documentación }\end{array}$} & \multirow{2}{*}{$\begin{array}{l}\text { Licenciados en } \\
\text { Filosofía y Letras } \\
\text { y/o Letras. } \\
\text { Licenciados en } \\
\text { Documentación }\end{array}$} & \\
\hline \multirow[t]{2}{*}{ 1995/97 } & $\begin{array}{l}\text { Información y } \\
\text { Documentación }\end{array}$ & & & \\
\hline & $\begin{array}{l}\text { Ciencias } \\
\text { Morfológicas y } \\
\text { Psicobiología }\end{array}$ & $\begin{array}{l}\text { Aparato Locomotor. } \\
\text { Valoración } \\
\text { Morfofuncional }\end{array}$ & $\begin{array}{l}\text { Licenciados en } \\
\text { Medicina y } \\
\text { Cirugía }\end{array}$ & $\begin{array}{l}\text { Doctor en } \\
\text { Medicina y } \\
\text { Cirugía }\end{array}$ \\
\hline $1996 / 98$ & $\begin{array}{l}\text { Información y } \\
\text { Documentación }\end{array}$ & \multirow{2}{*}{$\begin{array}{l}\text { Técnicas y Métodos } \\
\text { actuales en } \\
\text { Información y } \\
\text { Documentación }\end{array}$} & \multirow{2}{*}{$\begin{array}{l}\text { Licenciados en } \\
\text { Filosofía y Letras } \\
\text { y/o Letras. } \\
\text { Licenciados en } \\
\text { Documentación }\end{array}$} & \multirow{2}{*}{$\begin{array}{l}\text { Doctor en } \\
\text { Filosofía y } \\
\text { Letras (Filología } \\
\text { Hispánica) }\end{array}$} \\
\hline \multirow[t]{2}{*}{ 1997/99 } & $\begin{array}{l}\text { Información y } \\
\text { Documentación }\end{array}$ & & & \\
\hline & $\begin{array}{l}\text { Ciencias } \\
\text { Morfológicas y } \\
\text { Psicobiología }\end{array}$ & $\begin{array}{l}\text { Aparato Locomotor. } \\
\text { Valoración } \\
\text { Morfofuncional }\end{array}$ & $\begin{array}{l}\text { Licenciados en } \\
\text { Medicina y } \\
\text { Cirugía }\end{array}$ & $\begin{array}{l}\text { Doctor en } \\
\text { Medicina y } \\
\text { Cirugía }\end{array}$ \\
\hline \multirow[t]{2}{*}{$1998 / 00$} & $\begin{array}{l}\text { Información y } \\
\text { Documentación }\end{array}$ & $\begin{array}{l}\text { Técnicas y Métodos } \\
\text { actuales en } \\
\text { Información y } \\
\text { Documentación }\end{array}$ & $\begin{array}{l}\text { Licenciados en } \\
\text { Filosofía y Letras } \\
\text { y/o Letras. } \\
\text { Licenciados en } \\
\text { Documentación }\end{array}$ & $\begin{array}{l}\text { Doctor en } \\
\text { Filosofía y } \\
\text { Letras (Filología } \\
\text { Hispánica) }\end{array}$ \\
\hline & $\begin{array}{l}\text { Ciencias } \\
\text { Morfológicas y } \\
\text { Psicobiología }\end{array}$ & $\begin{array}{l}\text { Aparato Locomotor. } \\
\text { Valoración } \\
\text { Morfofuncional }\end{array}$ & $\begin{array}{l}\text { Licenciados en } \\
\text { Medicina y } \\
\text { Cirugía }\end{array}$ & $\begin{array}{l}\text { Doctor en } \\
\text { Medicina y } \\
\text { Cirugía }\end{array}$ \\
\hline
\end{tabular}

Scire. $7: 2$ (jul.-dic. 2001) 39-52. 


\section{Anexo II. Programas Doctorado al amparo del Real Decreto 778/1998}

\begin{tabular}{|c|c|c|c|}
\hline Bienio & Departamento & Programa & Procedencia Alumnos \\
\hline \multirow[t]{4}{*}{ 1999/01 } & $\begin{array}{l}\text { Información y } \\
\text { Documentación }\end{array}$ & $\begin{array}{l}\text { Técnicas y Métodos } \\
\text { actuales en Información } \\
\text { y Documentación * }\end{array}$ & $\begin{array}{l}\text { Licenciados en } \\
\text { Documentación, Periodismo, } \\
\text { Filosofía y Letras, Ingeniería } \\
\text { Informática, Comunicación } \\
\text { Audiovisual, Publicidad y } \\
\text { Relaciones Públicas }\end{array}$ \\
\hline & $\begin{array}{l}\text { Ciencias Morfológicas y } \\
\text { Psicobiología ** }\end{array}$ & $\begin{array}{l}\text { Neurociencia/Aparato } \\
\text { Locomotor }\end{array}$ & $\begin{array}{l}\text { Licenciados en Medicina y } \\
\text { Cirugía, Biología, Bioquímica, } \\
\text { Psicología, Farmacia, } \\
\text { Educación Física y Ciencias } \\
\text { del Deporte y Veterinaria }\end{array}$ \\
\hline & $\begin{array}{l}\text { Departamentos de: } \\
\text { Farmacología y } \\
\text { Fisiología, Biología } \\
\text { Celular, Bioquímica y } \\
\text { Biología Molecular "B" }\end{array}$ & $\begin{array}{l}\text { Ciencias Biomédicas } \\
\text { Experimentales }\end{array}$ & $\begin{array}{l}\text { Licenciados en Medicina y } \\
\text { Cirugía, Biología, Veterinaria, } \\
\text { Farmacia, Bioquímica, } \\
\text { Odontología, Ingeniería } \\
\text { Química, Educación Física e } \\
\text { Ingeniero Agrónomo }\end{array}$ \\
\hline & $\begin{array}{l}\text { Departamento de } \\
\text { Expresión Plástica, } \\
\text { Musical y Dinámica }\end{array}$ & $\begin{array}{l}\text { Actividad Física y } \\
\text { Deporte }\end{array}$ & $\begin{array}{l}\text { Licenciados en Educación } \\
\text { Física, Pedagogía, Psicología } \\
\text { y Medicina }\end{array}$ \\
\hline \multirow[t]{4}{*}{ 2000/02 } & $\begin{array}{l}\text { Información y } \\
\text { Documentación }\end{array}$ & $\begin{array}{l}\text { Técnicas y Métodos } \\
\text { actuales en Información } \\
\text { y Documentación * }\end{array}$ & $\begin{array}{l}\text { Licenciados en } \\
\text { Documentación, Periodismo, } \\
\text { Filosofía y Letras, Ingeniería } \\
\text { Informática, Comunicación } \\
\text { Audiovisual, Publicidad y } \\
\text { Relaciones Públicas }\end{array}$ \\
\hline & $\begin{array}{l}\text { Ciencias Morfológicas y } \\
\text { Psicobiología ** }\end{array}$ & $\begin{array}{l}\text { Neurociencia/Aparato } \\
\text { Locomotor }\end{array}$ & $\begin{array}{l}\text { Licenciados en Medicina y } \\
\text { Cirugía, Biología, Bioquímica, } \\
\text { Psicología, Farmacia, } \\
\text { Educación Física y Ciencias } \\
\text { del Deporte y Veterinaria }\end{array}$ \\
\hline & $\begin{array}{l}\text { Departamentos de: } \\
\text { Farmacología y } \\
\text { Fisiología, Biología } \\
\text { Celular, Bioquímica y } \\
\text { Biología Molecular "B" }\end{array}$ & $\begin{array}{l}\text { Ciencias Biomédicas } \\
\text { Experimentales }\end{array}$ & $\begin{array}{l}\text { Licenciados en Medicina y } \\
\text { Cirugía, Biología, Veterinaria, } \\
\text { Farmacia, Bioquímica, } \\
\text { Odontología, Ingeniería } \\
\text { Química, Educación Física e } \\
\text { Ingeniero Agrónomo }\end{array}$ \\
\hline & $\begin{array}{l}\text { Departamento de } \\
\text { Expresión Plástica, } \\
\text { Musical y Dinámica }\end{array}$ & $\begin{array}{l}\text { Actividad Física y } \\
\text { Deporte }\end{array}$ & $\begin{array}{l}\text { Licenciados en Educación } \\
\text { Física, Pedagogía, Psicología } \\
\text { y Medicina }\end{array}$ \\
\hline
\end{tabular}

* Programa Conjunto con el Departamento de Comunicación Audiovisual, Documentación e Historia del Arte, de la Universidad Politécnica de Valencia. La colaboración se inició en le bienio 1998/2000, aunque hasta el curso siguiente no se ofreció el programa interuniversitario.

** Programa Conjunto con el Dpto. de Psicología Experimental y Psicobiología, de la Universidad de Almería. 